Junshan Sun*

\title{
Crystal structure of bis $\left(\mu_{3}\right.$-oxido)-bis $\left(\mu_{2}-2,3,4,5\right.$-tetrafluorobenzoato- $\kappa^{2} 0: 0^{\prime}$ )-bis(2,3,4,5-tetrafluorobenzoato-kO)-oktakis(3-chlorobenzyl-kC) tetratin(IV), $\mathrm{C}_{84} \mathrm{H}_{52} \mathrm{Cl}_{8} \mathrm{~F}_{16} \mathrm{O}_{10} \mathrm{Sn}_{4}$
}

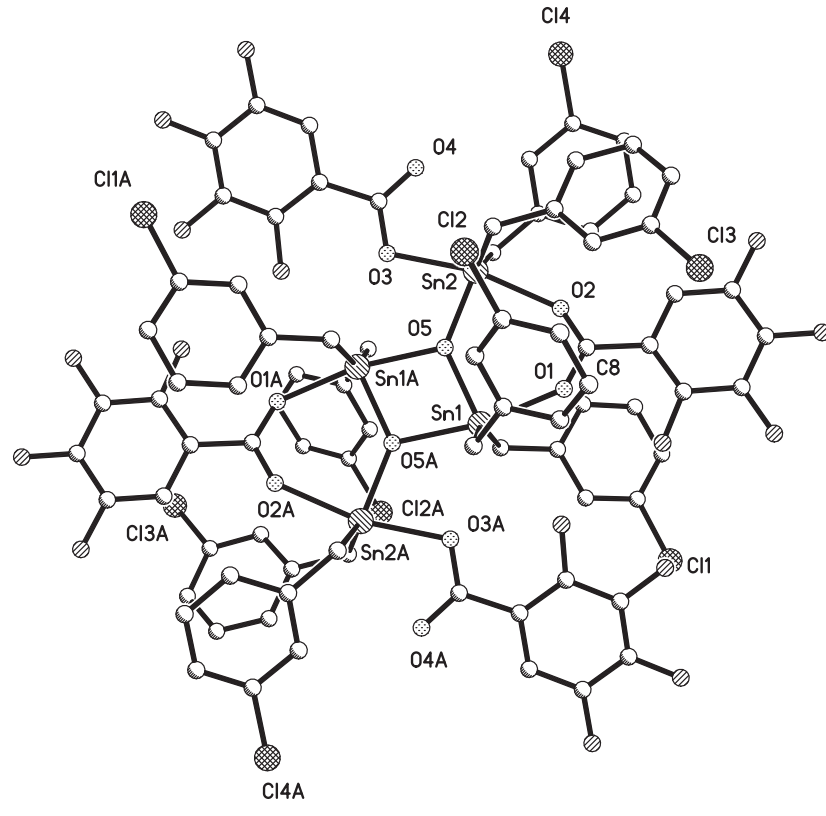

https://doi.org/10.1515/ncrs-2019-0613

Received August 24, 2019; accepted December 9, 2019; available online January 3, 2020

\section{Abstract \\ $\mathrm{C}_{84} \mathrm{H}_{52} \mathrm{Cl}_{8} \mathrm{~F}_{16} \mathrm{O}_{10} \mathrm{Sn}_{4}$, triclinic, $P \overline{1}$ (no. 2), $a=11.2999$ (9) $\AA$, $b=14.1471(11) \AA, \quad c=15.2378(12) \AA, \quad \alpha=67.656(1)^{\circ}$, $\beta=72.115(1)^{\circ}, \quad \gamma=69.730(1)^{\circ}, \quad V=2069.9(3) \AA^{3}, \quad Z=1$, $R_{\mathrm{gt}}(F)=0.0284, w R_{\text {ref }}\left(F^{2}\right)=0.0732, T=293(2) \mathrm{K}$.}

\section{CCDC no.: 1970757}

The molecular structure is shown in the figure. Table 1 contains crystallographic data and Table 2 contains the list of the atoms including atomic coordinates and displacement parameters.

\section{Source of material}

In a typical experiment, 2,3,4,5-tetrafluorobenzoic acid (0.194 g; $1 \mathrm{mmol}$ ) and 3-chlorobenzyltin oxide were dissolved in $18 \mathrm{~mL}$ benzene. This mixture was heated and refluxed to

\footnotetext{
${ }^{*}$ Corresponding author: Junshan Sun, Organic Photo-electric Laboratory, Depertment of Chemistry, College of Taishan, Tai'an 271099, Shandong Province, China, e-mail: sunhl0826@163.com. https://orcid.org/0000-0001-8031-228X
}

Table 1: Data collection and handling.

\begin{tabular}{ll}
\hline Crystal: & Colorless block \\
Size: & $0.19 \times 0.15 \times 0.11 \mathrm{~mm}$ \\
Wavelength: & Mo K $\alpha$ radiation $(0.71073 \AA$ A $)$ \\
$\mu:$ & $1.55 \mathrm{~mm}^{-1}$ \\
Diffractometer, scan mode: & Bruker SMART APEX-II, \\
$\theta_{\text {max }}$, completeness: & $25.0^{\circ}, 99 \%$ \\
$\mathrm{~N}(\mathrm{hkl})_{\text {measured }}, \mathrm{N}(\mathrm{hkl})_{\text {unique }}, R_{\text {int }}:$ & $14986,7212,0.022$ \\
Criterion for $\mathrm{I}_{\text {obs }}, \mathrm{N}(\mathrm{hkl})_{\mathrm{gt}}:$ & $\mathrm{I}_{\text {obs }}>2 \sigma\left(\mathrm{I}_{\text {obs }}\right), 6227$ \\
$N(\text { param })_{\text {refined }}:$ & 550 \\
Programs: & Bruker [1], SHELX [2, 3] \\
\hline
\end{tabular}

Table 2: Fractional atomic coordinates and isotropic or equivalent isotropic displacement parameters $\left(\AA^{2}\right)$.

\begin{tabular}{lrrrr}
\hline Atom & $\boldsymbol{x}$ & $\boldsymbol{y}$ & $\boldsymbol{Z}$ & $\boldsymbol{U}_{\text {iso }} \boldsymbol{U}_{\text {eq }}$ \\
\hline Sn1 & $0.88194(2)$ & $1.04167(2)$ & $0.58518(2)$ & $0.02752(7)$ \\
Sn2 & $0.97499(2)$ & $0.75343(2)$ & $0.60816(2)$ & $0.02951(7)$ \\
Cl1 & $0.27012(12)$ & $1.28299(14)$ & $0.66624(14)$ & $0.1067(5)$ \\
Cl2 & $1.28313(14)$ & $0.73452(11)$ & $0.84871(13)$ & $0.0989(5)$ \\
Cl3 & $0.79498(14)$ & $0.71756(13)$ & $1.05807(10)$ & $0.0931(4)$ \\
Cl4 & $0.91403(14)$ & $0.31245(10)$ & $0.74449(12)$ & $0.0914(4)$ \\
F1 & $1.3015(2)$ & $0.78975(16)$ & $0.31669(16)$ & $0.0558(6)$ \\
F2 & $1.5166(2)$ & $0.7292(2)$ & $0.19830(18)$ & $0.0741(8)$ \\
F3 & $1.6545(2)$ & $0.5237(2)$ & $0.23782(19)$ & $0.0810(8)$ \\
F4 & $1.5710(2)$ & $0.37789(18)$ & $0.39652(18)$ & $0.0743(8)$ \\
F5 & $0.5588(2)$ & $1.04105(18)$ & $0.82905(19)$ & $0.0712(7)$ \\
F6 & $0.3646(3)$ & $1.0030(2)$ & $0.9755(2)$ & $0.0894(9)$ \\
F7 & $0.3306(2)$ & $0.8056(2)$ & $1.06083(18)$ & $0.0802(8)$ \\
F8 & $0.4951(3)$ & $0.6449(2)$ & $0.99634(19)$ & $0.0778(8)$ \\
01 & $0.7793(2)$ & $0.95897(18)$ & $0.72419(16)$ & $0.0397(6)$ \\
O2 & $0.7888(2)$ & $0.80882(18)$ & $0.70410(16)$ & $0.0390(6)$ \\
03 & $1.1468(2)$ & $0.73686(17)$ & $0.49430(16)$ & $0.0396(6)$ \\
O4 & $1.1508(3)$ & $0.5676(2)$ & $0.5556(2)$ & $0.0584(8)$ \\
05 & $0.98889(19)$ & $0.90481(15)$ & $0.55332(15)$ & $0.0304(5)$ \\
C1 & $1.1960(3)$ & $0.6391(3)$ & $0.4965(3)$ & $0.0385(8)$ \\
C2 & $1.3175(3)$ & $0.6133(3)$ & $0.4237(2)$ & $0.0362(8)$ \\
C3 & $1.3634(3)$ & $0.6864(3)$ & $0.3411(3)$ & $0.0392(8)$ \\
C4 & $1.4759(3)$ & $0.6566(3)$ & $0.2784(3)$ & $0.0462(9)$ \\
C5 & $1.5455(4)$ & $0.5530(3)$ & $0.2980(3)$ & $0.0511(10)$ \\
C6 & $1.5005(4)$ & $0.4800(3)$ & $0.3792(3)$ & $0.0487(10)$ \\
C7 & $1.3879(3)$ & $0.5081(3)$ & $0.4410(3)$ & $0.0418(8)$ \\
H7 & 1.358249 & 0.456598 & 0.495054 & $0.050 *$ \\
C8 & $0.7416(3)$ & $0.8779(3)$ & $0.7472(2)$ & $0.0323(7)$ \\
C9 & $0.6312(3)$ & $0.8616(3)$ & $0.8318(2)$ & $0.0342(7)$ \\
C10 & $0.5487(4)$ & $0.9419(3)$ & $0.8675(3)$ & $0.0449(9)$ \\
C11 & $0.4464(4)$ & $0.9231(3)$ & $0.9436(3)$ & $0.0537(10)$ \\
C12 & $0.4301(4)$ & $0.8233(4)$ & $0.9864(3)$ & $0.0535(10)$ \\
C13 & $0.5128(4)$ & $0.7425(3)$ & $0.9524(3)$ & $0.0492(10)$ \\
C14 & $0.6125(3)$ & $0.7604(3)$ & $0.8749(3)$ & $0.0412(8)$
\end{tabular}


Table 2 (continued)

\begin{tabular}{|c|c|c|c|c|}
\hline Atom & $x$ & $y$ & $z$ & $U_{\text {iso }} \star / U_{\text {eq }}$ \\
\hline H14 & 0.667259 & 0.705221 & 0.851241 & $0.049^{\star}$ \\
\hline C15 & $0.7236(3)$ & $1.0904(3)$ & $0.5144(2)$ & $0.0394(8)$ \\
\hline $\mathrm{H} 15 \mathrm{~A}$ & 0.717304 & 1.163531 & 0.474594 & $0.047^{\star}$ \\
\hline H15B & 0.747221 & 1.049085 & 0.470487 & $0.047^{*}$ \\
\hline C16 & $0.5913(3)$ & $1.0842(3)$ & $0.5712(2)$ & $0.0369(8)$ \\
\hline C17 & $0.5018(3)$ & $1.1735(3)$ & $0.5918(3)$ & $0.0482(9)$ \\
\hline $\mathrm{H} 17$ & 0.524492 & 1.237029 & 0.569917 & $0.058^{\star}$ \\
\hline C18 & $0.3798(4)$ & $1.1682(4)$ & $0.6446(3)$ & $0.0630(12)$ \\
\hline C19 & $0.3428(4)$ & $1.0762(4)$ & $0.6776(3)$ & $0.0715(14)$ \\
\hline H19 & 0.260324 & 1.073605 & 0.713632 & $0.086^{*}$ \\
\hline $\mathrm{C} 20$ & $0.4290(5)$ & $0.9878(4)$ & $0.6568(4)$ & $0.0702(14)$ \\
\hline $\mathrm{H} 20$ & 0.404922 & 0.924864 & 0.678898 & $0.084^{\star}$ \\
\hline C21 & $0.5525(4)$ & $0.9918(3)$ & $0.6029(3)$ & $0.0509(10)$ \\
\hline $\mathrm{H} 21$ & 0.609564 & 0.931795 & 0.588058 & $0.061^{*}$ \\
\hline $\mathrm{C} 22$ & $0.9855(3)$ & $1.0750(3)$ & $0.6620(2)$ & $0.0370(8)$ \\
\hline $\mathrm{H} 22 \mathrm{~A}$ & 1.067124 & 1.084497 & 0.619577 & $0.044^{\star}$ \\
\hline $\mathrm{H} 22 \mathrm{~B}$ & 0.937243 & 1.142291 & 0.673449 & $0.044^{\star}$ \\
\hline $\mathrm{C} 23$ & $1.0129(3)$ & $0.9968(3)$ & $0.7562(2)$ & $0.0351(7)$ \\
\hline C24 & $1.1201(3)$ & $0.9109(3)$ & $0.7596(3)$ & $0.0411(8)$ \\
\hline $\mathrm{H} 24$ & 1.172842 & 0.899244 & 0.702395 & $0.049^{\star}$ \\
\hline $\mathrm{C} 25$ & $1.1492(4)$ & $0.8426(3)$ & $0.8475(3)$ & $0.0569(11)$ \\
\hline C26 & $1.0715(5)$ & $0.8562(4)$ & $0.9334(3)$ & $0.0716(14)$ \\
\hline $\mathrm{H} 26$ & 1.091929 & 0.809863 & 0.992387 & $0.086^{\star}$ \\
\hline $\mathrm{C} 27$ & $0.9636(5)$ & $0.9395(4)$ & $0.9305(3)$ & $0.0739(14)$ \\
\hline $\mathrm{H} 27$ & 0.909552 & 0.949782 & 0.987813 & $0.089^{\star}$ \\
\hline $\mathrm{C} 28$ & $0.9351(4)$ & $1.0076(4)$ & $0.8431(3)$ & $0.0564(11)$ \\
\hline $\mathrm{H} 28$ & 0.860697 & 1.063110 & 0.842463 & $0.068^{\star}$ \\
\hline C29 & $1.0688(3)$ & $0.6730(3)$ & $0.7282(3)$ & $0.0442(9)$ \\
\hline $\mathrm{H} 29 \mathrm{~A}$ & 1.139058 & 0.614522 & 0.713622 & $0.053^{\star}$ \\
\hline H29B & 1.105941 & 0.721327 & 0.735453 & $0.053^{*}$ \\
\hline C30 & $0.9846(3)$ & $0.6309(3)$ & $0.8225(2)$ & $0.0414(8)$ \\
\hline C31 & $0.9329(3)$ & $0.6862(3)$ & $0.8889(3)$ & $0.0453(9)$ \\
\hline H31 & 0.949667 & 0.750949 & 0.874588 & $0.054^{*}$ \\
\hline C32 & $0.8566(4)$ & $0.6463(4)$ & $0.9765(3)$ & $0.0559(10)$ \\
\hline C33 & $0.8289(4)$ & $0.5520(4)$ & $0.9990(3)$ & $0.0668(13)$ \\
\hline H33 & 0.777258 & 0.525612 & 1.058173 & $0.080^{\star}$ \\
\hline C34 & $0.8780(5)$ & $0.4974(4)$ & $0.9333(3)$ & $0.0681(13)$ \\
\hline H34 & 0.858673 & 0.433727 & 0.947682 & $0.082^{\star}$ \\
\hline C35 & $0.9559(4)$ & $0.5350(3)$ & $0.8459(3)$ & $0.0576(11)$ \\
\hline H35 & 0.989492 & 0.496097 & 0.802371 & $0.069^{\star}$ \\
\hline C36 & $0.8575(4)$ & $0.7103(3)$ & $0.5501(3)$ & $0.0441(9)$ \\
\hline $\mathrm{H} 36 \mathrm{~A}$ & 0.789231 & 0.771793 & 0.527486 & $0.053^{\star}$ \\
\hline H36B & 0.909412 & 0.685649 & 0.495383 & $0.053^{*}$ \\
\hline C37 & $0.8000(3)$ & $0.6249(3)$ & $0.6260(3)$ & $0.0428(9)$ \\
\hline C38 & $0.8704(4)$ & $0.5204(3)$ & $0.6445(3)$ & $0.0484(9)$ \\
\hline H38 & 0.950757 & 0.502073 & 0.605664 & $0.058^{\star}$ \\
\hline C39 & $0.8214(4)$ & $0.4428(3)$ & $0.7209(3)$ & $0.0535(10)$ \\
\hline C40 & $0.7035(5)$ & $0.4661(4)$ & $0.7788(3)$ & $0.0630(12)$ \\
\hline $\mathrm{H} 40$ & 0.673070 & 0.413113 & 0.830579 & $0.076^{\star}$ \\
\hline C41 & $0.6308(4)$ & $0.5686(4)$ & $0.7592(4)$ & $0.0681(13)$ \\
\hline H41 & 0.549015 & 0.585513 & 0.796854 & $0.082^{\star}$ \\
\hline C42 & $0.6784(4)$ & $0.6475(3)$ & $0.6835(3)$ & $0.0593(11)$ \\
\hline H42 & 0.627832 & 0.717033 & 0.670995 & $0.071^{\star}$ \\
\hline
\end{tabular}

$80{ }^{\circ} \mathrm{C}$, resulting in a colorless solution. The mixture was filtered and recrystallized. Crystals of the title compound were obtained by slow evaporation within one week.

\section{Experimental details}

All hydrogen atoms were geometrically positioned. Their $U_{\text {iso }}$ values were assigned with common isotropic displacement factors $U_{\text {iso }}(\mathrm{H})=1.2$ times $U_{\text {eq }}$.

\section{Comment}

In recent years, organotin(IV) complexes have been focused on acquiring well defined solid-state structures to learn the nature of their versatile coordination chemistry [4]. Moreover, they have received much attention because the tin(IV) is able to form stable bonds to get oligonuclear complexes [5-7].

The symmetric unit of the title structure contains two Sn centers with two carboxylate ligands, four chlorobenzyl moieties and one bridging $\mathrm{O}$ atom. Each $\mathrm{Sn}$ shows a fivecoordinated trigonal bipyramidal geometry. Sn1, 05, C22 and C15 occupy the equatorial planar positions and 01,05 are in apical positions. The bond angle of 01-Sn1-05 is $169.07(8)^{\circ}$, showing the distortion. Sn2, 05, C29 and C36 occupy the equatorial planar positions and $\mathrm{O} 2, \mathrm{O} 3$ are in apical positions. The bond angle of $02-\mathrm{Sn1}-\mathrm{O} 3$ is $167.00(8)^{\circ}$. The bond lengths of Sn1-01 is 2.179(2) $\AA$ and Sn2-02 is 2.240(2) $\AA$, which is close to the literature reported [8].

Acknowledgements: Financial support from the Shandong Provincial college Students Innovation and Entrepreneurship Training Program (No. 3003) are gratefully acknowledged.

\section{References}

1. BRUKER. SAINT, APEX2 and SADABS. Bruker AXS Inc., Madison, WI, USA (2009).

2. Sheldrick, G. M.: Crystal structure refinement with SHELXL. Acta Crystallogr. C71 (2015) 3-8.

3. Sheldrick, G. M.: SHELXT - integrated space-group and crystal-structure determination. Acta Crystallogr. A71 (2015) 3-8.

4. Pellerito, L.; Nagy, L.: Organotin(IV) complexes formed with biologically active ligands: equilibrium and structural studies, and some biological aspects Coord. Chem. Rev. 224 (2002) 111-150.

5. Ma, C. L.; Li, F.: Synthesis and crystal structures of three ladder distannoxane dimers $\left[\left(\mathrm{PhCH}_{2}\right)_{2}(\mathrm{Cl}) \mathrm{SnOSn}(\mathrm{X})\left(\mathrm{CH}_{2} \mathrm{Ph}\right)_{2}\right]_{2}(\mathrm{X}=\mathrm{Cl}$, OMe, OEt) Chin. J. Chem. 21 (2003) 146-152.

6. Prabusankar, G.; Jousseaume, B.; Toupance, T.; Allouchi, H.: Organic-inorganic $\mathrm{Sn}_{12}$ and organic $\mathrm{Sn}_{6}$ oxide-hydroxide clusters. Angew. Chem. Int. Ed. 45 (2006) 1255-1258.

7. Shankar, R.; Kumar, M.; Chadha, R. K.; Hundal, G.: Synthesis, characterization, and hydrolytic behavior of mixed-ligand diorganotin esters. Inorg. Chem. 42 (2003) 8585-8591.

8. Sun, J.; Qiu, L.; Zhang, R.; Ma, C.: Octabenzyl-di- $\mu_{3}-0 \times 0-$ bis- $\left(\mu-2,3,4,5\right.$-tetrafluoro-benzoato- $\left.\mathrm{K}^{2} \mathrm{O}: \mathrm{O}^{\prime}\right)$ bis $(2,3,4,5-$ tetrafluorobenzoato- $\mathrm{\kappa} O$ )tetratin(IV). Acta Crystallogr. E63 (2007) m62-m63. 